\title{
Cyclotomic Constructions of Cyclic Codes with Length Being the Product of Two Primes
}

\author{
Cunsheng Ding
}

\begin{abstract}
Cyclic codes are an interesting type of linear codes and have applications in communication and storage systems due to their efficient encoding and decoding algorithms. They have been studied for decades and a lot of progress has been made. In this paper, three types of generalized cyclotomy of order two and three classes of cyclic codes of length $n_{1} n_{2}$ and dimension $\left(n_{1} n_{2}+1\right) / 2$ are presented and analysed, where $n_{1}$ and $n_{2}$ are two distinct primes. Bounds on their minimum odd-like weight are also proved. The three constructions produce the best cyclic codes in certain cases.
\end{abstract}

\section{Index Terms}

Cyclic codes, cyclotomy, difference sets, duadic codes, linear codes.

\section{INTRODUCTION}

Let $q$ be a power of a prime. A linear $[n, k, \omega ; q]$ code is a $k$-dimensional subspace of $\operatorname{GF}(q)^{n}$ with minimum (Hamming) distance $\omega$. Let $A_{i}$ denote the number of codewords with Hamming weight $i$ in a code $\mathcal{C}$ of length $n$. The weight enumerator of $\mathcal{C}$ is defined by

$$
1+A_{1} x+A_{2} x^{2}+\cdots+A_{n} x^{n} .
$$

A linear $[n, k]$ code $\mathcal{C}$ over the finite field $\mathrm{GF}(q)$ is called cyclic if $\left(c_{0}, c_{1}, \cdots, c_{n-1}\right) \in \mathcal{C}$ implies $\left(c_{n-1}, c_{0}, c_{1}, \cdots, c_{n-2}\right) \in \mathcal{C}$. Let $\operatorname{gcd}(n, q)=1$. By identifying any vector $\left(c_{0}, c_{1}, \cdots, c_{n-1}\right) \in \operatorname{GF}(q)^{n}$ with

$$
c_{0}+c_{1} x+c_{2} x^{2}+\cdots+c_{n-1} x^{n-1} \in \mathrm{GF}(q)[x] /\left(x^{n}-1\right),
$$

any code $\mathcal{C}$ of length $n$ over $\operatorname{GF}(q)$ corresponds to a subset of $\operatorname{GF}(q)[x] /\left(x^{n}-1\right)$. The linear code $\mathcal{C}$ is cyclic if and only if the corresponding subset in $\mathrm{GF}(q)[x] /\left(x^{n}-1\right)$ is an ideal of the ring $\mathrm{GF}(q)[x] /\left(x^{n}-1\right)$.

Note that every ideal of $\operatorname{GF}(q)[x] /\left(x^{n}-1\right)$ is principal. Let $\mathcal{C}=(g(x))$ be a cyclic code. Then $g(x)$ is called the generator polynomial and $h(x)=\left(x^{n}-1\right) / g(x)$ is referred to as the parity-check polynomial of $\mathcal{C}$.

A vector $\left(c_{0}, c_{1}, \cdots, c_{n-1}\right) \in \mathrm{GF}(q)^{n}$ is said to be even-like if $\sum_{i=0}^{n-1} c_{i}=0$, and is odd-like otherwise. The minimum weight of the even-like codewords, respectively the odd-like codewords of a code is the minimum even-like weight, respectively the minimum odd-like weight of the code.

The error correcting capability of cyclic codes may not be as good as some other linear codes. However, cyclic codes have wide applications in storage and communication systems because they have efficient encoding and decoding algorithms [3], [10], [20], [21]. For example, Reed-Solomon codes have found important applications from deep-space communication to consumer electronics. They are prominently used in consumer electronics such as CDs, DVDs, Blu-ray Discs, in data transmission technologies such as DSL \& WiMAX, in broadcast systems such as DVB and ATSC, and in computer applications such as RAID 6 systems.

Cyclic codes have been studied for decades and a lot of progress has been made (see for example, [1], [22], [11], [12], [16], [17], [19], [23]). However, the total number of cyclic codes of length $n$ and dimension $k$ over a finite field $\mathrm{GF}(q)$ is in general unknown, not to mention the construction of all of

C. Ding is with the Department of Computer Science and Engineering, The Hong Kong University of Science and Technology, Clear Water Bay, Kowloon, Hong Kong. Email: cding@ust.hk 
them. An important problem in studying cyclic codes is to find a simple construction of the best cyclic codes.

In this paper, three types of generalized cyclotomy of order two are described and three simple constructions of cyclic codes of length $n_{1} n_{2}$ and dimension $\left(n_{1} n_{2}+1\right) / 2$ are presented, where $n_{1}$ and $n_{2}$ are two distinct primes. Bounds on their minimum odd-like weight are also proved. Some of the codes in this paper are among the best cyclic codes. This is the main motivation of investigating the three cyclotomic constructions of the cyclic codes in this paper.

\section{All CyCliC CODES With PARAMETERS $\left[n_{1} n_{2},\left(n_{1} n_{2}+1\right) / 2 ; q\right]$}

Throughout this paper let $n_{1}$ and $n_{2}$ be two distinct odd primes, $n=n_{1} n_{2}$, and let $q$ be a power of a prime such that $\operatorname{gcd}(q, n)=1$. We also define

$$
N=\operatorname{ord}_{n}(q)=\operatorname{lcm}\left(\operatorname{ord}_{n_{1}}(q), \operatorname{ord}_{n_{2}}(q)\right) .
$$

In this paper we define $\theta=\alpha^{\left(q^{N}-1\right) / n}$, where $\alpha$ is a generator of $\operatorname{GF}\left(q^{N}\right)^{*}$. Hence $\theta$ is an $n$th primitive root of unity in $\operatorname{GF}\left(q^{N}\right)^{*}$.

\section{A. The general case}

Let $S_{0}^{(n)}$ be the subgroup of $\mathbf{Z}_{n}^{*}$ generated by $q$. Then the cardinality of $S_{0}^{(n)}$ is $N$. Let $S_{i}^{(n)}$ be all the cosets of the subgroup $S_{0}^{(n)}$, where $0 \leq i<\left(n_{1}-1\right)\left(n_{2}-1\right) / N$. Define

$$
F_{j}^{(n)}(x)=\prod_{i \in S_{j}^{(n)}}\left(x-\theta^{i}\right)
$$

It is well known that all $F_{j}^{(n)}(x)$ are irreducible polynomials over $\operatorname{GF}(q)$ and have degree $N$.

Similarly, for each $j \in\{1,2\}$ let $S_{0}^{\left(n_{j}\right)}$ be the subgroup of $\mathbf{Z}_{n_{j}}^{*}$ generated by $q$. Then the cardinality of $S_{0}^{\left(n_{j}\right)}$ is $\operatorname{ord}_{n_{j}}(q)$. Let $S_{i}^{\left(n_{j}\right)}$ be all the cosets of the subgroup $S_{0}^{\left(n_{j}\right)}$, where $0 \leq i<\left(n_{j}-1\right) / \operatorname{ord}_{n_{j}}(q)$. Let $\theta_{j}=\alpha^{\left(q^{N}-1\right) / n_{j}}$. Define

$$
F_{i}^{\left(n_{j}\right)}(x)=\prod_{h \in S_{i}^{\left(n_{j}\right)}}\left(x-\theta_{j}^{h}\right)
$$

It is also well known that all $F_{i}^{\left(n_{j}\right)}(x)$ are irreducible polynomials over $\operatorname{GF}(q)$ and have degree $\operatorname{ord}_{n_{j}}(q)$. In addition, we have

$$
x^{n_{j}}-1=(x-1) \prod_{i=0}^{\frac{n_{j}-1}{\operatorname{ord}_{j}(q)}-1} F_{i}^{\left(n_{j}\right)}(x)
$$

for each $j \in\{1,2\}$.

Summarizing the discussions above, we have

$$
\prod_{i=0}^{\frac{n_{1}-1}{\operatorname{ord} n_{1}(q)}} F_{i}^{\left(n_{1}\right)}(x) \prod_{i=0}^{\frac{n_{2}-1}{\operatorname{ord} n_{2}(q)}-1} F_{i}^{\left(n_{2}\right)}(x) \prod_{i=0}^{\frac{\left(n_{1}-1\right)\left(n_{2}-1\right)}{\operatorname{ord} n_{2}(q)}-1} F_{i}^{(n)}(x) .
$$

It is hard to give a specific formula for the total number of $[n,(n+1) / 2 ; q]$ cyclic codes. However, this number is at least

$$
\left(\begin{array}{c}
\frac{n_{1}-1}{\operatorname{ord}_{n_{1}}(q)} \\
\frac{n_{1}-1}{\operatorname{ord}_{n_{1}}(q)}
\end{array}\right)\left(\begin{array}{c}
\frac{n_{2}-1}{\operatorname{ord}_{n_{2}}(q)} \\
\frac{n_{2}-1}{2 \operatorname{ord}_{n_{2}}(q)}
\end{array}\right)\left(\begin{array}{l}
\frac{\left(n_{1}-1\right)\left(n_{2}-1\right)}{\operatorname{ord}_{n}(q)} \\
\frac{\left(n_{1}-1\right)\left(n_{2}-1\right)}{2 \operatorname{ord}_{n}(q)}
\end{array}\right),
$$

provided that $\left(n_{j}-1\right) / \operatorname{ord}_{n_{j}}(q)$ is even for all $j$. In many cases, this is indeed the exact number of $[n,(n+1) / 2 ; q]$ cyclic codes.

In order to show that some of the codes constructed in this paper are the best cyclic codes, we provide information about all binary cyclic codes of length 119 and dimension 60, and all quaternary codes of length 35 and dimension 18 in the next three subsections. 


\section{B. All binary cyclic codes with length 119 and dimension 60}

We now consider all binary cyclic codes of length 119 and dimension 60. Note that the factorization of $x^{119}-1$ over $\mathrm{GF}(2)$ is

$$
x^{119}-1=f_{1}(x) f_{31}(x) f_{32}(x) f_{81}(x) f_{82}(x) f_{241}(x) f_{242}(x) f_{243}(x) f_{244}(x),
$$

where the irreducible polynomials

$$
\begin{aligned}
f_{1}(x) & =x+1 \\
f_{31}(x) & =x^{3}+x+1 \\
f_{32}(x) & =x^{3}+x^{2}+1 \\
f_{81}(x) & =x^{8}+x^{5}+x^{4}+x^{3}+1 \\
f_{82}(x) & =x^{8}+x^{7}+x^{6}+x^{4}+x^{2}+x+1 \\
f_{241}(x) & =x^{24}+x^{20}+x^{18}+x^{17}+x^{12}+x^{11}+x^{9}+x^{7}+x^{5}+x^{3}+1, \\
f_{242}(x) & =x^{24}+x^{21}+x^{19}+x^{17}+x^{15}+x^{13}+x^{12}+x^{7}+x^{6}+x^{4}+1, \\
f_{243}(x) & =x^{24}+x^{22}+x^{20}+x^{14}+x^{12}+x^{11}+x^{9}+x^{8}+x^{7}+x^{5}+x^{2}+x+1, \\
f_{244}(x) & =x^{24}+x^{23}+x^{22}+x^{19}+x^{17}+x^{16}+x^{15}+x^{13}+x^{12}+x^{10}+x^{4}+x^{2}+1 .
\end{aligned}
$$

Hence, there are altogether 24 binary cyclic codes of length 119 and dimension 60 . Their generator polynomials and minimum nonzero weights are described in Table [.

TABLE I

ALL BINARY CYCLIC CODES OF LENGTH 119 AND DIMENSION 60

\begin{tabular}{|l|r|}
\hline Generator Polynomial & Minimum Weight \\
\hline \hline$f_{31} f_{81} f_{241} f_{242}$ & 6 \\
\hline$f_{31} f_{81} f_{241} f_{243}$ & 11 \\
\hline$f_{31} f_{81} f_{241} f_{244}$ & 8 \\
\hline$f_{31} f_{81} f_{242} f_{243}$ & 4 \\
\hline$f_{31} f_{81} f_{242} f_{244}$ & 12 \\
\hline$f_{31} f_{81} f_{243} f_{244}$ & 12 \\
\hline$f_{31} f_{82} f_{241} f_{242}$ & 12 \\
\hline$f_{31} f_{82} f_{241} f_{243}$ & 12 \\
\hline$f_{31} f_{82} f_{241} f_{244}$ & 8 \\
\hline$f_{31} f_{82} f_{242} f_{243}$ & 4 \\
\hline$f_{31} f_{82} f_{242} f_{244}$ & 11 \\
\hline$f_{31} f_{82} f_{243} f_{244}$ & 6 \\
\hline$f_{32} f_{81} f_{241} f_{242}$ & 6 \\
\hline$f_{32} f_{81} f_{241} f_{243}$ & 12 \\
\hline$f_{32} f_{81} f_{241} f_{244}$ & 4 \\
\hline$f_{32} f_{81} f_{242} f_{243}$ & 8 \\
\hline$f_{32} f_{81} f_{242} f_{244}$ & 11 \\
\hline$f_{32} f_{81} f_{243} f_{244}$ & 12 \\
\hline$f_{32} f_{82} f_{241} f_{242}$ & 12 \\
\hline$f_{32} f_{82} f_{241} f_{243}$ & 11 \\
\hline$f_{32} f_{82} f_{241} f_{244}$ & 4 \\
\hline$f_{32} f_{82} f_{242} f_{243}$ & 8 \\
\hline$f_{32} f_{82} f_{242} f_{244}$ & 12 \\
\hline$f_{32} f_{82} f_{243} f_{244}$ & 6 \\
\hline
\end{tabular}

C. All ternary cyclic codes with length 143 and dimension 72

Let $q=3, n_{1}=11$ and $n_{2}=13$. We have

$$
\operatorname{ord}_{n_{1}}(q)=5, \operatorname{ord}_{n_{2}}(q)=3, \operatorname{ord}_{n}(q)=15 .
$$


The polynomial $x^{143}-1$ is factorized into the product of the following irreducible polynomials:

$$
\begin{aligned}
& x+2, \\
& x^{3}+2 x+2, \\
& x^{3}+x^{2}+2, \\
& x^{3}+x^{2}+x+2, \\
& x^{3}+2 x^{2}+2 x+2, \\
& x^{5}+2 x^{3}+x^{2}+2 x+2, \\
& x^{5}+x^{4}+2 x^{3}+x^{2}+2, \\
& x^{15}+x^{12}+x^{9}+2 x^{8}+x^{7}+x^{6}+x^{5}+2 x^{4}+2 x^{3}+x^{2}+x+2, \\
& x^{15}+x^{12}+2 x^{11}+x^{10}+2 x^{8}+2 x^{6}+2 x^{5}+x^{4}+x^{3}+x^{2}+2, \\
& x^{15}+x^{13}+2 x^{11}+x^{8}+2 x^{6}+x^{5}+2 x^{4}+x^{2}+x+2, \\
& x^{15}+x^{13}+2 x^{12}+2 x^{11}+2 x^{10}+x^{8}+x^{7}+x^{6}+x^{5}+x^{2}+2 x+2, \\
& x^{15}+2 x^{13}+2 x^{12}+2 x^{11}+x^{10}+x^{9}+x^{7}+2 x^{5}+x^{4}+2 x^{3}+2, \\
& x^{15}+x^{14}+2 x^{13}+2 x^{10}+2 x^{9}+2 x^{8}+2 x^{7}+x^{5}+x^{4}+x^{3}+2 x^{2}+2, \\
& x^{15}+2 x^{14}+2 x^{13}+x^{11}+2 x^{10}+x^{9}+2 x^{7}+x^{4}+2 x^{2}+2, \\
& x^{15}+2 x^{14}+2 x^{13}+x^{12}+x^{11}+2 x^{10}+2 x^{9}+2 x^{8}+x^{7}+2 x^{6}+2 x^{3}+2 .
\end{aligned}
$$

The total number of $[143,72 ; 3]$ cyclic codes is thus

$$
\left(\begin{array}{l}
2 \\
1
\end{array}\right)\left(\begin{array}{l}
4 \\
2
\end{array}\right)\left(\begin{array}{l}
8 \\
4
\end{array}\right)=840
$$

Due to the limitation of our computational power, we have not been able to compute the minimum weights of the 840 ternary cyclic codes of length 143 and dimension 72 .

\section{All quaternary cyclic codes with length 35 and dimension 18}

We now consider all quaternary cyclic codes of length 35 and dimension 18. Let $w$ be a generator of $\mathrm{GF}\left(2^{2}\right)$ such that $w^{2}+w+1=0$. Then the factorization of $x^{35}-1$ over $\mathrm{GF}(4)$ is

$$
x^{35}-1=f_{1}(x) f_{21}(x) f_{22}(x) f_{31}(x) f_{32}(x) f_{61}(x) f_{62}(x) f_{63}(x) f_{64}(x),
$$

where the irreducible polynomials

$$
\begin{aligned}
f_{1}(x) & =x+1 \\
f_{21}(x) & =x^{2}+w x+1, \\
f_{22}(x) & =x^{2}+w^{2} x+1, \\
f_{31}(x) & =x^{3}+x+1, \\
f_{32}(x) & =x^{3}+x^{2}+1, \\
f_{61}(x) & =x^{6}+w x^{4}+w x^{3}+x^{2}+w^{2} x+1, \\
f_{62}(x) & =x^{6}+w^{2} x^{4}+w^{2} x^{3}+x^{2}+w x+1, \\
f_{63}(x) & =x^{6}+w x^{5}+x^{4}+w^{2} x^{3}+w^{2} x^{2}+1, \\
f_{64}(x) & =x^{6}+w^{2} x^{5}+x^{4}+w x^{3}+w x^{2}+1 .
\end{aligned}
$$

Hence, there are altogether 24 quaternary cyclic codes of length 35 and dimension 18 . Their generator polynomials and minimum nonzero weights are described in Table 【. 
TABLE II

ALL QUATERNARY CYCLIC CODES OF LENGTH 35 AND DIMENSION 18

\begin{tabular}{|l|r|}
\hline Generator Polynomial & Minimum Weight \\
\hline \hline$f_{21} f_{31} f_{61} f_{62}$ & 4 \\
\hline$f_{21} f_{31} f_{61} f_{63}$ & 7 \\
\hline$f_{21} f_{31} f_{61} f_{64}$ & 8 \\
\hline$f_{21} f_{31} f_{62} f_{63}$ & 4 \\
\hline$f_{21} f_{31} f_{62} f_{64}$ & 8 \\
\hline$f_{21} f_{31} f_{63} f_{64}$ & 7 \\
\hline$f_{21} f_{32} f_{61} f_{62}$ & 7 \\
\hline$f_{21} f_{32} f_{61} f_{63}$ & 8 \\
\hline$f_{21} f_{32} f_{61} f_{64}$ & 8 \\
\hline$f_{21} f_{32} f_{62} f_{63}$ & 4 \\
\hline$f_{21} f_{32} f_{62} f_{64}$ & 7 \\
\hline$f_{21} f_{32} f_{63} f_{64}$ & 4 \\
\hline$f_{22} f_{31} f_{61} f_{62}$ & 4 \\
\hline$f_{22} f_{31} f_{61} f_{63}$ & 8 \\
\hline$f_{22} f_{31} f_{61} f_{64}$ & 4 \\
\hline$f_{22} f_{31} f_{62} f_{63}$ & 8 \\
\hline$f_{22} f_{31} f_{62} f_{64}$ & 7 \\
\hline$f_{22} f_{31} f_{63} f_{64}$ & 7 \\
\hline$f_{22} f_{32} f_{61} f_{62}$ & 7 \\
\hline$f_{22} f_{32} f_{61} f_{63}$ & 7 \\
\hline$f_{22} f_{32} f_{61} f_{64}$ & 4 \\
\hline$f_{22} f_{32} f_{62} f_{63}$ & 8 \\
\hline$f_{22} f_{32} f_{62} f_{64}$ & 8 \\
\hline$f_{22} f_{32} f_{63} f_{64}$ & 4 \\
\hline
\end{tabular}

\section{A CYCLOTOMY OF ORDER TWO AND ITS CODES}

\section{A. A generalization of Whiteman's cyclotomy of order two}

Let $d=\operatorname{gcd}\left(n_{1}-1, n_{2}-1\right)$, and let $g_{1}$ and $g_{2}$ be a primitive root of $n_{1}$ and $n_{2}$ respectively. Define $g$ by

$$
g \equiv g_{1} \quad\left(\bmod n_{1}\right), \quad g \equiv g_{2} \quad\left(\bmod n_{2}\right) .
$$

By the Chinese Remainder Theorem, $g$ is unique modulo $n$ and a common primitive root of $n_{1}$ and $n_{2}$, and $\operatorname{ord}_{n}(g)=\left(n_{1}-1\right)\left(n_{2}-1\right) / d=e$.

The following proposition is proved in [24].

Proposition 1: Define $\nu$ by

$$
\nu \equiv g \quad\left(\bmod n_{1}\right), \quad \nu \equiv 1 \quad\left(\bmod n_{2}\right) .
$$

Then

$$
\mathbf{Z}_{n}^{*}=\left\{g^{s} \nu^{i}: s=0,1, \cdots, e-1 ; i=0,1, \cdots, d-1\right\} .
$$

Whiteman's generalized cyclotomic classes $W_{i}$ of order $d$ are defined by

$$
W_{i}=\left\{g^{s} \nu^{i}: s=0,1, \cdots, e-1\right\}, i=0,1, \cdots, d-1 .
$$

Clearly, $d$ is even. Define two subsets $U_{0}$ and $U_{1}$ of $\mathbf{Z}_{n}^{*}$ with the cyclotomic classes $W_{i}$ of order $d$ as

$$
U_{0}=\bigcup_{i=0}^{(d-2) / 2} W_{2 i} \text { and } U_{1}=\bigcup_{i=0}^{(d-2) / 2} W_{2 i+1} .
$$

Note that $U_{0}$ is a subgroup of $\mathbf{Z}_{n}^{*}$ and $U_{1}=\nu U_{0}$, which is a coset of $U_{0}$. The sets $U_{0}$ and $U_{1}$ form a new cyclotomy of order 2, which is different from Whiteman's when $d>2$ and coincides with Whiteman's cyclotomy of order 2 when $d=2$. 
Whiteman proved that $U_{0} \cup\left\{0, n_{2}, 2 n_{2}, \cdots,\left(n_{1}-1\right) n_{2}\right\}$ is a difference set when $n_{2}=n_{1}+2$, i.e., when they are twin primes [24]. This is the well-known twin-prime difference set which has applications in combinatorics, coding theory and communication systems.

The following proposition is proved in [24].

Proposition 2: Let symbols be the same as before. Then

$$
-1=\left\{\begin{array}{l}
g^{e / 2} \text { when }\left(n_{1}-1\right)\left(n_{2}-1\right) / d^{2} \text { is odd } \\
g^{t} \nu^{d / 2} \text { when }\left(n_{1}-1\right)\left(n_{2}-1\right) / d^{2} \text { is even, }
\end{array}\right.
$$

where $t$ is some integer with $0 \leq t \leq e-1$.

B. The eight cyclic codes from the cyclotomy $\left(U_{0}, U_{1}\right)$

Let $D_{0}^{\left(n_{i}\right)}$ and $D_{1}^{\left(n_{i}\right)}$ be the set of quadratic residues and nonresidues modulo $n_{i}$ respectively. Define for each $i \in\{0,1\}$

$$
d_{i}^{\left(n_{1}\right)}(x)=\prod_{j \in D_{i}^{\left(n_{1}\right)}}\left(x-\theta^{n_{2} j}\right), d_{i}^{\left(n_{2}\right)}(x)=\prod_{j \in D_{i}^{\left(n_{2}\right)}}\left(x-\theta^{n_{1} j}\right) .
$$

By definition,

$$
x^{n_{j}}-1=(x-1) d_{0}^{\left(n_{j}\right)}(x) d_{1}^{\left(n_{j}\right)}(x) .
$$

We define

$$
u_{j}(x)=\prod_{i \in U_{j}}\left(x-\theta^{i}\right), \quad j=0,1
$$

and

$$
u(x)=u_{0}(x) u_{1}(x) .
$$

Obviously, $u(x) \in \mathrm{GF}(q)[x]$. In fact, we have

$$
x^{n}-1=\prod_{i=0}^{n-1}\left(x-\theta^{i}\right)=\frac{\left(x^{n_{1}}-1\right)\left(x^{n_{2}}-1\right) u(x)}{x-1} .
$$

Proposition 3: If $q \in U_{0}$ and $q \bmod n_{i} \in D_{0}^{\left(n_{i}\right)}$ for each $i \in\{1,2\}$, we have $u_{i}(x) \in \operatorname{GF}(q)[x]$ and $d_{i}^{\left(n_{j}\right)}(x) \in \mathrm{GF}(q)[x]$, and

$$
x^{n}-1=(x-1) d_{0}^{\left(n_{1}\right)}(x) d_{0}^{\left(n_{2}\right)}(x) u_{0}(x) d_{1}^{\left(n_{1}\right)}(x) d_{1}^{\left(n_{2}\right)}(x) u_{1}(x) .
$$

Proof: Assume that $q \in U_{0}$. By Proposition 9, $q U_{i}=U_{i}$ for each $i$. It then follows that

$$
u_{j}(x)^{q}=\prod_{i \in U_{j}}\left(x^{q}-\theta^{q i}\right)=\prod_{i \in q U_{j}}\left(x^{q}-\theta^{i}\right)=\prod_{i \in U_{j}}\left(x^{q}-\theta^{i}\right)=u_{j}\left(x^{q}\right) .
$$

Similarly, one can prove that $d_{i}^{\left(n_{j}\right)}(x) \in \mathrm{GF}(q)[x]$ for each $j$. The desired equality then follows from the definitions of these polynomials.

Under the conditions that $q \in U_{0}$ and $q \bmod n_{i} \in D_{0}^{\left(n_{i}\right)}$ for each $i \in\{1,2\}$, let $\mathcal{U}_{(i, j, h)}^{\left(n_{1}, n_{2}, q\right)}$ denote the cyclic code over $\operatorname{GF}(q)$ with generator polynomial $u_{i}(x) d_{j}^{\left(n_{1}\right)}(x) d_{h}^{\left(n_{2}\right)}(x)$, where $(i, j, h) \in\{0,1\}^{3}$. The eight codes clearly have length $n$ and dimension $(n+1) / 2$.

Proposition 4: There is no $\ell \in U_{1}$ such that $\ell \bmod n_{i} \in D_{1}^{\left(n_{i}\right)}$ for all $i \in\{1,2\}$.

Proof: Let $\ell=g^{s} \nu^{i} \in U_{1}$ for some $s$ and $i$ with $0 \leq s \leq e-1$ and $0 \leq i \leq d-1$. Then $i$ is odd and

$$
\ell \equiv g^{s+i} \quad\left(\bmod n_{1}\right) \text { and } \ell \equiv g^{s} \quad\left(\bmod n_{2}\right)
$$

Since $i$ is odd, it is impossible to have $\ell \bmod n_{i} \in D_{1}^{\left(n_{i}\right)}$ for all $i \in\{1,2\}$ at the same time. 
Proposition 4 means that it is impossible to prove a square-root bound on the minimum odd-like weight of the codes with the traditional argument for the square-root bound of quadratic residue codes. Hence, we develop another type of bound.

Proposition 5: Let $\omega_{(i, j, h)}^{(n)}$ denote the minimum odd-like weight of the code $\mathcal{U}_{(i, j, h)}^{\left(n_{1}, n_{2}, q\right)}$ and let $\omega_{i}^{\left(n / n_{j}\right)}$ denote the minimum odd-like weight of the cyclic code of length $n$ over $\operatorname{GF}(q)$ generated by the polynomial $\left(x^{n}-1\right) /(x-1) d_{i}^{\left(n_{j}\right)}(x)$ for all $i \in\{0,1\}$ and all $j \in\{0,1\}$. We have then

$$
\omega_{(0,0,0)}^{(n)} \geq \sqrt{\max \left(\omega_{1}^{\left(n / n_{1}\right)}, \omega_{1}^{\left(n / n_{2}\right)}\right)} .
$$

Proof: Let $\ell=g^{s} x^{i}$ for some $s$ and $i$. Note that

$$
\ell \equiv g^{s+i} \quad\left(\bmod n_{1}\right) \text { and } \ell \equiv g^{s} \quad\left(\bmod n_{2}\right)
$$

We have then the following conclusions.

1) There is an $\ell_{1} \in U_{1}$ such that $\ell_{1} \bmod n_{1} \in D_{0}^{\left(n_{1}\right)}$ and $\ell_{1} \bmod n_{2} \in D_{1}^{\left(n_{1}\right)}$.

2) There is an $\ell_{2} \in U_{1}$ such that $\ell_{2} \bmod n_{1} \in D_{1}^{\left(n_{1}\right)}$ and $\ell_{2} \bmod n_{2} \in D_{0}^{\left(n_{1}\right)}$.

Let $a(x)$ be a codeword in $\mathcal{U}_{(0,0,0)}^{\left(n_{1}, n_{2}, q\right)}$ with minimum odd-like weight $\omega_{(0,0,0)}^{(n)}$. Then $a\left(x^{\ell_{1}}\right)$ is a codeword in $\mathcal{U}_{(1,0,1)}^{\left(n_{1}, n_{2}, q\right)}$ with minimum odd-like weight $\omega_{(0,0,0)}^{(n)}$, and $a\left(x^{\ell_{2}}\right)$ is a codeword in $\mathcal{U}_{(1,1,0)}^{\left(n_{1}, n_{2}, q\right)}$ with minimum odd-like weight $\omega_{(0,0,0)}^{(n)}$. It follows that $a(x) a\left(x^{\ell_{1}}\right)$ is an odd-like codeword in the cyclic code with the generator polynomial $\left(x^{n}-1\right) /(x-1) d_{1}^{\left(n_{1}\right)}(x)$ and $a(x) a\left(x^{\ell_{2}}\right)$ is an odd-like codeword in the cyclic code with the generator polynomial $\left(x^{n}-1\right) /(x-1) d_{1}^{\left(n_{2}\right)}(x)$. Since $a(x) a\left(x^{\ell_{1}}\right)$ and $a(x) a\left(x^{\ell_{2}}\right)$ have at most $\left(\omega_{(0,0,0)}^{(n)}\right)^{2}$ terms, the desired lower bound then follows.

Similar bounds for other $\omega_{(i, j, h)}^{(n)}$ can be written down. The lower bound of Proposition 5 depends on the minimum odd-like weight of two special codes and may not be convenient to use. It will be seen later that the $\mathrm{BCH}$ bound on these codes could be much better.

We have the following remarks on Whiteman's cyclotomy of order two and its codes defined above.

1) In [6] Ding and Helleseth generalized Whiteman's cyclotomy of order two into the case that $n=$ $\prod_{i=1}^{t} n_{i}^{e_{i}}$, where all $n_{i}$ are pairwise distinct primes and $\operatorname{gcd}\left(n_{i}-1, n_{j}-1\right)=2$ for all pairs of distinct $i$ and $j$, and introduced eight binary cyclotomic codes.

2) However, in the case that $n=n_{1} n_{2}$ the cyclotomy of order two in [6] is just a special case of Whiteman's cyclotomy of order two because of the required condition $\operatorname{gcd}\left(n_{1}-1, n_{2}-1\right)=2$. Hence the cyclotomic codes defined in [6] are special cases of the eight codes over GF $(q)$ in this section when $q=2$ and $\operatorname{gcd}\left(n_{1}-1, n_{2}-1\right)=2$. So Whiteman's cyclotomy of order two yields more codes. For example, when $\left(n_{1}, n_{2}\right)=(17,41)$ Whiteman's cyclotomy of order two gives eight binary cyclic codes, while the cyclotomy introduced in [6] does not work for this pair of $n_{1}$ and $n_{2}$.

\section{The binary case}

Proposition 6: The integer $2 \in U_{0}$ if and only if

$$
n_{1} \equiv \pm 1 \quad(\bmod 8) \text { and } n_{2} \equiv \pm 1 \quad(\bmod 8)
$$

or

$$
n_{1} \equiv \pm 3 \quad(\bmod 8) \text { and } n_{2} \equiv \pm 3 \quad(\bmod 8) .
$$

Proof: Recall that $\mathbf{Z}_{n}^{*}=U_{0} \cup U_{1}$. By Proposition 1, there are two integers $0 \leq s \leq e-1$ and $0 \leq i \leq d-1$ such that $2=g^{s} \nu^{i}$. It then follows from (11) that

$$
g^{s+i} \equiv 2 \quad\left(\bmod n_{1}\right) \text { and } g^{s} \equiv 2 \quad\left(\bmod n_{2}\right) .
$$


Hence, $i$ is even if and only if the one of the conditions in this proposition is satisfied. Note that $2 \in U_{0}$ if and only if $i$ is even. The proof is then completed.

Therefore, in the case that $n_{1} \equiv \pm 1 \quad(\bmod 8)$ and $n_{2} \equiv \pm 1 \quad(\bmod 8)$, we have indeed the eight binary cyclic codes $\mathcal{U}_{(i, j, h)}^{\left(n_{1}, n_{2}, 2\right)}$.

Example 1: When $\left(n_{1}, n_{2}, q\right)=(7,17,2)$, we have

$$
\begin{gathered}
U_{0}=\left\{\begin{array}{l}
1,2,3,4,5,6,8,9,10,12,15,16,18,20,24,25,27,30,31,32,36,40,41,43,45,48, \\
50,53,54,60,61,62,64,67,72,73,75,80,81,82,86,90,93,96,97,100,106,108
\end{array}\right\}, \\
U_{1}=\left\{\begin{array}{l}
11,13,19,22,23,26,29,33,37,38,39,44,46,47,52,55,57,58,59,65,66,69,71,74,76,78, \\
79,83,87,88,89,92,94,95,99,101,103,104,107,109,110,111,113,114,115,116,117,118
\end{array}\right\}
\end{gathered}
$$

and

$$
\begin{aligned}
d_{0}^{\left(n_{1}\right)}(x)= & x^{3}+x+1, \\
d_{1}^{\left(n_{1}\right)}(x)= & x^{3}+x^{2}+1, \\
d_{0}^{\left(n_{2}\right)}(x)= & x^{8}+x^{7}+x^{6}+x^{4}+x^{2}+x+1, \\
d_{1}^{\left(n_{2}\right)}(x)= & x^{8}+x^{5}+x^{4}+x^{3}+1, \\
u_{0}(x)= & x^{48}+x^{46}+x^{41}+x^{39}+x^{37}+x^{33}+x^{30}+x^{29}+x^{27}+x^{25}+x^{23}+x^{18}+x^{17}+x^{13}+ \\
& x^{10}+x^{9}+x^{8}+x^{7}+x^{6}+x^{5}+x^{4}+x^{3}+x^{2}+x+1, \\
u_{1}(x)= & x^{48}+x^{47}+x^{46}+x^{45}+x^{44}+x^{43}+x^{42}+x^{41}+x^{40}+x^{39}+x^{38}+x^{35}+x^{31}+x^{30}+ \\
& x^{25}+x^{23}+x^{21}+x^{19}+x^{18}+x^{15}+x^{11}+x^{9}+x^{7}+x^{2}+1 .
\end{aligned}
$$

The minimum nonzero weights of the eight codes are given in Table IIII. Four of the eight codes are the best binary cyclic codes of length 119 and dimension 60 and have minimum weight 12 according to Table I] and the remaining four have minimum weight 11.

TABLE III

THE BINARY CYCLIC CODES OF LENGTH 119 AND DIMENSION 60 FROM WHITEMAN'S CYCLOTOMY

\begin{tabular}{|l|r|}
\hline The code & Minimum Weight \\
\hline \hline $\mathcal{U}_{(0,0,0)}^{(7,17,2)}$ & 12 \\
\hline $\mathcal{U}_{(1,0,0)}^{(7,17,2)}$ & 11 \\
\hline $\mathcal{U}_{(0,1,0)}^{(7,17,2)}$ & 11 \\
\hline $\mathcal{U}_{(0,0,1)}^{(7,17,2)}$ & 11 \\
\hline $\mathcal{U}_{(1,1,0)}^{(7,17,2)}$ & 12 \\
\hline $\mathcal{U}_{(1,0,1)}^{(7,17,2)}$ & 12 \\
\hline $\mathcal{U}_{(0,1,1)}^{(7,17,2)}$ & 12 \\
\hline $\mathcal{U}_{(1,1,1)}^{(7,17,2)}$ & 11 \\
\hline
\end{tabular}

\section{The ternary case}

Proposition 7: The integer $3 \in U_{0}$ and $3 \bmod n_{i} \in D_{0}^{\left(n_{i}\right)}$ for all $i$ if and only if

$$
n_{1} \equiv \pm 1 \quad(\bmod 12) \text { and } n_{2} \equiv \pm 1 \quad(\bmod 12) .
$$

Proof: With the Law of Quadratic Reciprocity, one can prove that $3 \bmod n_{i} \in D_{0}^{\left(n_{i}\right)}$ if and only if $n_{i} \equiv \pm 1 \quad(\bmod 12)$. In addition, let $3=g^{s} \nu^{i}$ for some $s$ and $i$. Then we have

$$
3 \equiv g^{s+i} \quad\left(\bmod n_{1}\right) \text { and } 3 \equiv g^{s} \quad\left(\bmod n_{2}\right) .
$$


If 3 is a quadratic residue modulo both $n_{1}$ and $n_{2}$, both $s$ and $s+i$ must be even. It follows that $i$ must be even. Hence, $3 \in U_{0}$.

So in the case that $n_{1} \equiv \pm 1 \quad(\bmod 12)$ and $n_{2} \equiv \pm 1 \quad(\bmod 12)$, we have indeed the eight ternary cyclic codes $\mathcal{U}_{(i, j, h)}^{\left(n_{1}, n_{2}, 3\right)}$.

Example 2: When $\left(n_{1}, n_{2}, q\right)=(11,13,3)$, the minimum nonzero weights of the eight ternary codes are given in Table IV] Four of the eight codes have minimum weight 12, and the remaining four have minimum weight 11 .

TABLE IV

THE TERNARY CYCLIC CODES OF LENGTH 143 AND DIMENSION 72 FROM WHITEMAN'S CYCLOTOMY

\begin{tabular}{|l|r|}
\hline \multicolumn{1}{|c|}{ The code } & Minimum Weight \\
\hline \hline $\mathcal{U}_{(0,0,0)}^{(11,13,3)}$ & 12 \\
\hline $\mathcal{U}_{(1,0,0)}^{(11,13,3)}$ & 11 \\
\hline $\mathcal{U}_{(0,1,0)}^{(11,13)}$ & 11 \\
\hline $\mathcal{U}_{(0,0,1)}^{(11,13,3)}$ & 11 \\
\hline $\mathcal{U}_{(1,1,0)}^{(11,13,3)}$ & 12 \\
\hline $\mathcal{U}_{(1,0,1)}^{(11,13)}$ & 12 \\
\hline $\mathcal{U}_{(0,1,1)}^{(11,13,3)}$ & 12 \\
\hline $\mathcal{U}_{(1,1,1)}^{(11,13,3)}$ & 11 \\
\hline
\end{tabular}

\section{E. The quaternary case}

The following proposition can be similarly proved.

Proposition 8: The integer $4 \in U_{0}$ and $4 \bmod n_{j} \in D_{0}^{\left(n_{j}\right)}$ for all $j$ if and only if

$$
n_{1} \equiv \pm 1 \quad(\bmod 4) \text { and } n_{2} \equiv \pm 1 \quad(\bmod 4) \text {. }
$$

Therefore, in the case that $n_{1} \equiv \pm 1 \quad(\bmod 4)$ and $n_{2} \equiv \pm 1 \quad(\bmod 4)$, we have indeed the eight quaternary cyclic codes $\mathcal{U}_{(i, j, h)}^{\left(n_{1}, n_{2}, 4\right)}$.

Example 3: When $\left(n_{1}, n_{2}, q\right)=(5,7,4)$, the minimum nonzero weights of the eight quaternary codes are given in Table $\mathrm{V}$. Four of the eight codes are the best quaternary cyclic codes of length 35 and dimension 18 and have minimum weight 8 according to Table $\amalg$ and the remaining four have minimum weight 7 .

TABLE V

THE QUATERNARY CYCLIC CODES OF LENGTH 35 AND DIMENSION 18 FROM WHITEMAN'S CYCLOTOMY

\begin{tabular}{|l|r|}
\hline The code & Minimum Weight \\
\hline \hline $\mathcal{U}_{(0,0,0)}^{(5,7,4)}$ & 8 \\
\hline $\mathcal{U}_{(1,0,0)}^{(5,7,4)}$ & 7 \\
\hline $\mathcal{U}_{(0,1,0)}^{(5,7,4)}$ & 7 \\
\hline $\mathcal{U}_{(0,0,1)}^{(5,7,4)}$ & 7 \\
\hline $\mathcal{U}_{(1,1,0)}^{(5,7,4)}$ & 8 \\
\hline $\mathcal{U}_{(1,0,1)}^{(5,7,4)}$ & 8 \\
\hline $\mathcal{U}_{(0,1,1)}^{(5,7,4)}$ & 8 \\
\hline $\mathcal{U}_{(1,1,1)}^{(5,7,4)}$ & 7 \\
\hline
\end{tabular}




\section{F. The robustness of Whiteman's cyclotomy of order two}

All the binary cyclic codes of length 119 and dimension 60 and all the quaternary cyclic codes of length 35 and dimension 18 presented before are either the best or almost the best in terms of their minimum weights. In this section, we will provide theoretical evidences for this fact. We consider only the case that $\left(n_{1}, n_{2}\right)=(7,17)$.

Let $\left(n_{1}, n_{2}, q\right)=(7,17, q)$, and let $I(i, j, h)$ denote the set of zeros $\theta^{l}$ of the generator polynomial of the code $\mathcal{U}_{(i, j, h)}^{(7,17, q)}$. Then the set of exponents $l$ of $\theta^{l}$ in $I(i, j, h)$ is the following.

- When $(i, j, h)=(0,0,0)$, the set is

$$
\left\{\begin{array}{l}
1,2,3,4,5,6,7,8,9,10,12,14,15,16,17,18,20,24,25,27,28,30, \\
31,32,34,36,40,41,43,45,48,50,53,54,56,60,61,62,63,64,67 \\
68,72,73,75,80,81,82,86,90,91,93,96,97,100,105,106,108,112
\end{array}\right\} .
$$

The $\mathrm{BCH}$ bound says that the minimum weight of the code is at least 11 . The case that $(i, j, h)=$ $(1,1,1)$ is equivalent to this case as any element in $D_{1}$ times this set gives the set for the case $(i, j, h)=(1,1,1)$. So we have the same lower bound for the code $\mathcal{U}_{(1,1,1)}^{(7,17, q)}$.

- When $(i, j, h)=(1,0,0)$, the set is

$$
\left\{\begin{array}{l}
7,11,13,14,17,19,22,23,26,28,29,33,34,37,38,39,44,46,47,52,55, \\
56,57,58,59,63,65,66,68,69,71,74,76,78,79,83,87,88,89,91,92,94, \\
95,99,101,103,104,105,107,109,110,111,112,113,114,115,116,117,118
\end{array}\right\} .
$$

The $\mathrm{BCH}$ bound says that the minimum weight of the code is at least 11 . The case that $(i, j, h)=$ $(0,1,1)$ is equivalent to this case. So we have the same lower bound for the code $\mathcal{U}_{(0,1,1)}^{(7,17, q)}$.

- When $(i, j, h)=(0,1,0)$, the set is

$$
\left\{\begin{array}{l}
1,2,3,4,5,6,7,8,9,10,12,14,15,16,18,20,24,25,27,28,30,31,32,36, \\
40,41,43,45,48,50,51,53,54,56,60,61,62,63,64,67,72,73,75,80,81, \\
82,85,86,90,91,93,96,97,100,102,105,106,108,112
\end{array}\right\} .
$$

The $\mathrm{BCH}$ bound says that the minimum weight of the code is at least 11 . The case that $(i, j, h)=$ $(1,0,1)$ is equivalent to this case. So we have the same lower bound for the code $\mathcal{U}_{(1,0,1)}^{(7,17, q)}$.

- When $(i, j, h)=(0,0,1)$, the set is

$$
\left\{\begin{array}{l}
1,2,3,4,5,6,8,9,10,12,15,16,17,18,20,21,24,25,27,30,31,32,34,35, \\
36,40,41,42,43,45,48,49,50,53,54,60,61,62,64,67,68,70,72,73,75,77, \\
80,81,82,84,86,90,93,96,97,98,100,106,108
\end{array}\right\} .
$$

The $\mathrm{BCH}$ bound says that the minimum weight of the code is at least 11 . The case that $(i, j, h)=$ $(1,1,0)$ is equivalent to this case. So we have the same lower bound for the code $\mathcal{U}_{(1,1,0)}^{(7,17, q)}$.

The lower bound 11 is true not only for $q=2$, but also for $q=4$. This experimental data explains why the codes are the best or almost the best among all cyclic codes of the same length and the same dimension.

\section{AN EXTENSION OF AN EARLIER GENERALIZED CYCLOTOMY OF ORDER TWO AND ITS CODES}

\section{A. The extension of an earlier generalized cyclotomy of order two}

Since $n_{1}$ and $n_{2}$ are odd primes, $d$ must be even. It is easily seen that $e=\left(n_{1}-1\right)\left(n_{2}-1\right) / d$ is also even. Define

$$
\begin{aligned}
& D_{0}=\left\{g^{2 s} \nu^{i}: s=0,1, \cdots,(e-2) / 2 ; i=0,1, \cdots, d-1\right\} \\
& D_{1}=\left\{g^{2 s+1} \nu^{i}: s=0,1, \cdots,(e-2) / 2 ; i=0,1, \cdots, d-1\right\} .
\end{aligned}
$$


Clearly, $D_{1}=g D_{0}$, and $D_{0}$ and $D_{1}$ form a partition of $\mathbf{Z}_{n}^{*}$.

A proof of the following proposition is straightforward and is omitted.

Proposition 9: Let symbols be the same as before.

1) $D_{0}$ is a subgroup of $\mathbf{Z}_{n}^{*}$ and has order $\left(n_{1}-1\right)\left(n_{2}-1\right) / 2$.

2) If $a \in D_{0}$, we have $a D_{i}=D_{i}$. If $a \in D_{1}$, we have $a D_{i}=D_{(i+1) \bmod 2}$.

The sets $D_{0}$ and $D_{1}$ are the cyclotomic classes of order 2, and are clearly different from Whiteman's cyclotomic classes of order 2 described in Section III. We point out here that this generalized cyclotomy of order two is the same as the one introduced by Ding and Helleseth when $\operatorname{gcd}\left(n_{1}-1, n_{2}-1\right)=2$, and is indeed an extension. We will use $D_{0}$ and $D_{1}$ to describe cyclic codes in the sequel.

\section{B. The construction of eight cyclic codes}

Let $\theta$ and other symbols be the same as before. Define

$$
d_{i}(x)=\prod_{j \in D_{i}}\left(x-\theta^{j}\right)
$$

Proposition 10: If $q \in D_{0}$ and $q \bmod n_{i} \in D_{0}^{\left(n_{i}\right)}$ for each $i \in\{1,2\}$, we have $d_{i}(x) \in \mathrm{GF}(q)[x]$ and $d_{i}^{\left(n_{j}\right)}(x) \in \mathrm{GF}(q)[x]$, and

$$
x^{n}-1=(x-1) d_{0}^{\left(n_{1}\right)}(x) d_{0}^{\left(n_{2}\right)}(x) d_{0}(x) d_{1}^{\left(n_{1}\right)}(x) d_{1}^{\left(n_{2}\right)}(x) d_{1}(x) .
$$

Proof: The proof of Proposition 3 is easily modified into a proof for this proposition.

Under the conditions that $q \in D_{0}$ and $q \bmod n_{i} \in D_{0}^{\left(n_{i}\right)}$ for each $i \in\{1,2\}$, let $\mathcal{D}_{(i, j, h)}^{\left(n_{1}, n_{2}, q\right)}$ denote the cyclic code over $\operatorname{GF}(q)$ with generator polynomial $d_{i}(x) d_{j}^{\left(n_{1}\right)}(x) d_{h}^{\left(n_{2}\right)}(x)$, where $(i, j, h) \in\{0,1\}^{3}$. The eight codes clearly have length $n$ and dimension $(n+1) / 2$.

Proposition 11: There is an $\ell \in U_{1}$ such that $\ell \bmod n_{i} \in D_{1}^{\left(n_{i}\right)}$ for all $i \in\{1,2\}$.

Proof: Take any $\ell=g^{s} \nu^{i} \in U_{1}$ for any odd $s$ and even $i$ with $0 \leq s \leq e-1$ and $0 \leq i \leq d-1$. Note that $i$ is even and

$$
\ell \equiv g^{s+i} \quad\left(\bmod n_{1}\right) \text { and } \ell \equiv g^{s} \quad\left(\bmod n_{2}\right)
$$

We have that $\ell \bmod n_{i} \in D_{1}^{\left(n_{i}\right)}$ for all $i \in\{1,2\}$ at the same time.

Due to Proposition 11 we can prove a square-root bound on the minimum odd-like weight of the codes.

Theorem 12: For each $(i, j, h) \in\{0,1\}^{3}$ the code $\mathcal{D}_{(i, j, h)}^{\left(n_{1}, n_{2}, q\right)}$ has parameters $[n,(n+1) / 2]$. Let $d_{(i, j, h)}$ denote the minimum odd-like weight in $\mathcal{D}_{(i, j, h)}^{\left(n_{1}, n_{2}, q\right)}$. Then

- $d_{(i, j, h)} \geq \sqrt{n}$, and

- $d_{(i, j, h)}^{2}-d_{(i, j, h)}+1 \geq n$ if $n_{1} \equiv-1 \quad(\bmod 8)$ and $n_{2} \equiv-1 \quad(\bmod 8)$.

Proof: We first prove that $\mathcal{D}_{(i, j, h)}^{\left(n_{1}, n_{2}, q\right)}$ and $\mathcal{D}_{((i+1) \bmod 2,(j+1) \bmod 2,(h+1) \bmod 2)}^{\left(n_{1}, n_{2}, q\right)}$ are equivalent. Note that $g \in D_{1}$ and $g$ is a quadratic nonresidue modulo both $n_{1}$ and $n_{2}$. Let $\ell \in D_{1}$ such that $\ell$ is a quadratic nonresidue modulo both $n_{1}$ and $n_{2}$. The permutation of coordinates in $\operatorname{GF}(2)[x] /\left(x^{n}-1\right)$ induced by

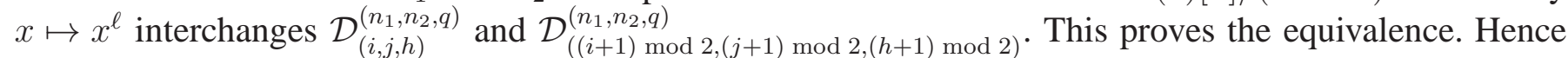
the two codes have the same minimum nonzero weight and the same minimum odd-like weight.

Let $a(x)$ be a codeword of minimum odd-like weight $d_{(i, j, h)}$ in $\mathcal{D}_{(i, j, h)}^{\left(n_{1}, n_{2}, q\right)}$. Hence, $\hat{a}(x)=a\left(x^{\ell}\right)$ is a codeword of minimum odd-like weight $d_{(i, j, h)}$ in $\mathcal{D}_{((i+1) \bmod 2,(j+1) \bmod 2,(h+1) \bmod 2)}^{\left(n_{1}, n_{2},\right)}$. Then $a(x) \hat{a}(x)$ must be in $\mathcal{D}_{(i, j, h)}^{\left(n_{1}, n_{2}, q\right)} \cap \mathcal{D}_{((i+1) \bmod 2,(j+1) \bmod 2,(h+1) \bmod 2)}^{\left(n_{1}, n_{2}, q\right)}$, i.e., is a multiple of

$$
\frac{x^{n}-1}{x-1}=x^{n-1}+x^{n-2}+\cdots+x+1 .
$$

Thus, $a(x) \hat{a}(x)$ has weight $n$. Since $a(x)$ has weight $d_{(i, j, h)}$, the maximum number of coefficients in $a(x) \hat{a}(x)$ is $d_{(i, j, h)}^{2}$. Therefore, $d_{(i, j, h)}^{2} \geq n$. 
If $n_{1} \equiv-1 \quad(\bmod 8)$ and $n_{2} \equiv-1 \quad(\bmod 8)$, by Proposition 2 we can take $\ell=-1$. In this case, the maximum number of coefficients in $a(x) \hat{a}(x)$ is $d_{(i, j, h)}^{2}-d_{(i, j, h)}^{2}+1$. Therefore, $d_{(i, j, h)}^{2}-d_{(i, j, h)}^{2}+1 \geq n$.

Note that the cyclotomy of order two described in this section is an extension of the one given in [5]. The codes described in [5] are only special cases of the codes of this section. First of all, the codes in [5] require the condition that $\operatorname{gcd}\left(n_{1}-1, n_{2}-1\right)=2$, while the eight codes in this section do not require this condition. Secondly, the codes in [5] are binary only, while the eight codes in this section are over $\mathrm{GF}(q)$.

\section{The binary case}

The following proposition will be useful later.

Proposition 13: Assume that $n_{1} \equiv \pm 1 \quad(\bmod 8)$ and $n_{2} \equiv \pm 1 \quad(\bmod 8)$. Then $-1 \in D_{1}$ if and only if $n_{2} \equiv-1 \quad(\bmod 8)$.

Proof: Let $-1=g^{s} \nu^{i}$ for some fixed $0 \leq s \leq e-1$ and $0 \leq i \leq d-1$. By (2),

$$
-1 \equiv g^{s+i} \quad\left(\bmod n_{1}\right), \quad-1 \equiv g^{s} \quad\left(\bmod n_{2}\right) .
$$

Hence $-1 \in D_{1}$ if and only if $s$ is odd, which is equivalent to $n_{2} \equiv-1 \quad(\bmod 8)$.

We will need the following proposition in the sequel.

Proposition 14: The integer $2 \in D_{0}$ if and only if $n_{2} \equiv \pm 1 \quad(\bmod 8)$.

Proof: Recall that $\mathbf{Z}_{n}^{*}=D_{0} \cup D_{1}$. By Proposition 1, there are two integers $0 \leq s \leq e-1$ and $0 \leq i \leq d-1$ such that $2=g^{s} \nu^{i}$. It then follows from (1) that

$$
g^{s+i} \equiv 2 \quad\left(\bmod n_{1}\right) \text { and } g^{s} \equiv 2 \quad\left(\bmod n_{2}\right) .
$$

Hence, $s$ is even if and only if $n_{2} \equiv \pm 1 \quad(\bmod 8)$. Note that $2 \in D_{0}$ if and only if $s$ is even. The proof is then completed.

If $n_{1} \equiv \pm 1 \quad(\bmod 8)$ and $n_{2} \equiv \pm 1 \quad(\bmod 8)$, then we have the following factorization of $x^{n}-1$ over $\operatorname{GF}(2)$ :

$$
x^{n}-1=(x-1) d_{0}^{\left(n_{1}\right)}(x) d_{0}^{\left(n_{2}\right)}(x) d_{0}(x) d_{1}^{\left(n_{1}\right)}(x) d_{1}^{\left(n_{2}\right)}(x) d_{1}(x),
$$

and thus the eight binary cyclic codes $\mathcal{D}_{(i, j, h)}^{\left(n_{1}, n_{2}, 2\right)}$.

Example 4: Let $\left(n_{1}, n_{2}, q\right)=(7,17,2)$. In this case we have

$$
\begin{aligned}
D_{0} & =\left\{\begin{array}{l}
1,2,4,8,9,13,15,16,18,19,25,26,30,32,33,36,38,43,47,50,52,53,55,59,60,64,66, \\
67,69,72,76,81,83,86,87,89,93,94,100,101,103,104,106,110,111,115,117,118
\end{array}\right\}, \\
D_{1} & =\left\{\begin{array}{l}
3,5,6,10,11,12,20,22,23,24,27,29,31,37,39,40,41,44,45,46,48,54,57,58,61,62, \\
65,71,73,74,75,78,79,80,82,88,90,92,95,96,97,99,107,108,109,113,114,116
\end{array}\right\}
\end{aligned}
$$

and

$$
\begin{aligned}
d_{0}^{\left(n_{1}\right)}(x)= & x^{3}+x+1, \\
d_{1}^{\left(n_{1}\right)}(x)= & x^{3}+x^{2}+1, \\
d_{0}^{\left(n_{2}\right)}(x)= & x^{8}+x^{7}+x^{6}+x^{4}+x^{2}+x+1, \\
d_{1}^{\left(n_{2}\right)}(x)= & x^{8}+x^{5}+x^{4}+x^{3}+1, \\
d_{0}(x)= & x^{48}+x^{45}+x^{44}+x^{43}+x^{42}+x^{41}+x^{39}+x^{38}+x^{37}+x^{36}+x^{35}+x^{34}+x^{32}+x^{30}+ \\
& x^{29}+x^{27}+x^{26}+x^{24}+x^{22}+x^{21}+x^{19}+x^{18}+x^{16}+x^{14}+x^{13}+x^{12}+x^{11}+x^{10}+ \\
& x^{9}+x^{7}+x^{6}+x^{5}+x^{4}+x^{3}+1, \\
d_{1}(x)= & x^{48}+x^{47}+x^{45}+x^{42}+x^{40}+x^{39}+x^{31}+x^{30}+x^{28}+x^{27}+x^{26}+x^{25}+x^{24}+x^{23}+ \\
& x^{22}+x^{21}+x^{20}+x^{18}+x^{17}+x^{9}+x^{8}+x^{6}+x^{3}+x+1 .
\end{aligned}
$$

The eight binary cyclic codes and their minimum weights are depicted in Table VI. In this example, half

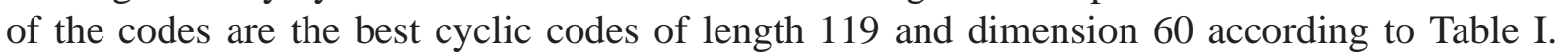


TABLE VI

THE BINARY CYCLIC CODES OF LENGTH 119 AND DIMENSION 60 FROM THE CYCLOTOMY OF SECTIONIV-A

\begin{tabular}{|l|r|}
\hline Generator Polynomial & Minimum Weight \\
\hline \hline $\mathcal{D}_{(0,0,0)}^{(7,17,2)}$ & 12 \\
\hline $\mathcal{D}_{(1,17,0)}^{(7,17)}$ & 6 \\
\hline $\mathcal{D}_{(0,1,0)}^{(7,17,2)}$ & 12 \\
\hline $\mathcal{D}_{(0,0,1)}^{(7,17,2)}$ & 6 \\
\hline $\mathcal{D}_{(1,1,0)}^{(7,7,2)}$ & 6 \\
\hline $\mathcal{D}_{(1,0,1)}^{(7,17,2)}$ & 12 \\
\hline $\mathcal{D}_{(0,1,1)}^{(7,17,2)}$ & 6 \\
\hline $\mathcal{D}_{(1,1,1)}^{(7,7,2)}$ & 12 \\
\hline
\end{tabular}

\section{The ternary case}

The proof of Proposition 7 can be slightly modified into a proof of the following.

Proposition 15: The integer $3 \in D_{0}$ and $3 \bmod n_{i} \in D_{0}^{\left(n_{i}\right)}$ for all $i$ if and only if

$$
n_{1} \equiv \pm 1 \quad(\bmod 12) \text { and } n_{2} \equiv \pm 1 \quad(\bmod 12) \text {. }
$$

Hence, in the case that $n_{1} \equiv \pm 1 \quad(\bmod 12)$ and $n_{2} \equiv \pm 1 \quad(\bmod 12)$, we have indeed the eight ternary cyclic codes $\mathcal{D}_{(i, j, h)}^{\left(n_{1}, n_{2}, 3\right)}$.

Example 5: When $\left(n_{1}, n_{2}, q\right)=(11,13,3)$, the minimum nonzero weights of the eight ternary codes are given in Table VII Four of the eight codes have minimum weight 12, and the remaining four have minimum weight 6 .

TABLE VII

THE TERNARY CYCLIC CODES OF LENGTH 143 AND DIMENSION 72 FROM THE CYCLOTOMY OF SECTIONIV-A

\begin{tabular}{|l|r|}
\hline The code & Minimum Weight \\
\hline \hline $\mathcal{D}_{(, 0,0)}^{(11,13,3)}$ & 12 \\
\hline $\mathcal{D}_{(1,0,0)}^{(11,13,3)}$ & 6 \\
\hline $\mathcal{D}_{(0,1,0)}^{(11,13,3)}$ & 12 \\
\hline $\mathcal{D}_{(0,0,1)}^{(1,13,3)}$ & 6 \\
\hline $\mathcal{D}_{(1,1,0)}^{(11,13,3)}$ & 6 \\
\hline $\mathcal{D}_{(1,0,1)}^{(11,13,3)}$ & 12 \\
\hline $\mathcal{D}_{(0,1,1)}^{(1,13,3)}$ & 6 \\
\hline $\mathcal{D}_{(1,1,1)}^{(11,13,3)}$ & 12 \\
\hline
\end{tabular}

\section{E. The quaternary case}

The following proposition can be similarly proved.

Proposition 16: Assume that $n_{1} \equiv-1 \quad(\bmod 4)$ and $n_{2} \equiv-1 \quad(\bmod 4)$. Then $-1 \in D_{1}$ and $-1 \in$ $D_{1}^{\left(n_{j}\right)}$ for all $j$.

We will in the sequel need the following proposition whose proof is omitted.

Proposition 17: The integer $4 \in D_{0}$ and $4 \bmod n_{j} \in D_{0}^{\left(n_{j}\right)}$ for all $j$ if and only if $n_{2} \equiv \pm 1 \quad(\bmod 4)$ for all $j$.

If $n_{1} \equiv \pm 1 \quad(\bmod 4)$ and $n_{2} \equiv \pm 1 \quad(\bmod 4)$, then we have the following factorization of $x^{n}-1$ over $\mathrm{GF}(4)$ :

$$
x^{n}-1=(x-1) d_{0}^{\left(n_{1}\right)}(x) d_{0}^{\left(n_{2}\right)}(x) d_{0}(x) d_{1}^{\left(n_{1}\right)}(x) d_{1}^{\left(n_{2}\right)}(x) d_{1}(x)
$$


and thus the eight quaternary cyclic codes $\mathcal{D}_{(i, j, h)}^{\left(n_{1}, n_{2}, 4\right)}$.

Example 6: Let $\left(n_{1}, n_{2}, q\right)=(5,7,4)$. In this case the eight quaternary cyclic codes and their minimum weights are depicted in Table VIII. In this example, half of the codes have minimum weight 7 and are almost the best quaternary cyclic codes of length 35 and dimension 18 according to Table II

TABLE VIII

THE QUATERNARY CYCLIC CODES OF LENGTH 35 AND DIMENSION 18 FROM THE CYCLOTOMY OF SECTIONIV-A

\begin{tabular}{|l|r|}
\hline Generator Polynomial & Minimum Weight \\
\hline \hline $\mathcal{D}_{(0,0,0)}^{(5,7,4)}$ & 7 \\
\hline $\mathcal{D}_{(1,0,0)}^{(5,7,4)}$ & 4 \\
\hline $\mathcal{D}_{(0,1,0)}^{(5,7)}$ & 7 \\
\hline $\mathcal{D}_{(0,0,1)}^{(5,7,4)}$ & 4 \\
\hline $\mathcal{D}_{(1,1,0)}^{(5,4)}$ & 4 \\
\hline $\mathcal{D}_{(1,0,1)}^{(5,7,4)}$ & 7 \\
\hline $\mathcal{D}_{(0,1,1)}^{(5,7,4)}$ & 4 \\
\hline $\mathcal{D}_{(1,1,1)}^{(5,7)}$ & 7 \\
\hline
\end{tabular}

\section{A NEW CYCLOTOMY OF ORDER TWO AND ITS CODES}

\section{A. The new cyclotomy of order two}

Let symbols be the same as before. Since $n_{1}$ and $n_{2}$ are odd primes, $d$ must be even. It is easily seen that $e=\left(n_{1}-1\right)\left(n_{2}-1\right) / d$ is also even. Define

$$
\begin{aligned}
& V_{0}=\left\{g^{s} \nu^{i}: 0 \leq s \leq e-1,0 \leq i \leq d-1 \text { and } s+i \text { is even }\right\}, \\
& V_{1}=\left\{g^{s} \nu^{i}: 0 \leq s \leq e-1,0 \leq i \leq d-1 \text { and } s+i \text { is odd }\right\} .
\end{aligned}
$$

Clearly, $V_{1}=g V_{0}$, and $V_{0}$ and $V_{1}$ form a partition of $\mathbf{Z}_{n}^{*}$.

A proof of the following proposition is straightforward and is omitted.

Proposition 18: Let symbols be the same as before.

1) $V_{0}$ is a subgroup of $\mathbf{Z}_{n}^{*}$ and has order $\left(n_{1}-1\right)\left(n_{2}-1\right) / 2$.

2) If $a \in V_{0}$, we have $a V_{i}=V_{i}$. If $a \in V_{1}$, we have $a V_{i}=V_{(i+1) \bmod 2}$.

The sets $V_{0}$ and $V_{1}$ are the new cyclotomic classes of order 2, and are clearly different from Whiteman's cyclotomic classes of order 2 described in Section $\amalg$ and the one of Section IV-A.

\section{B. The construction of eight cyclic codes}

Let $\theta$ and other symbols be the same as before. Define

$$
v_{i}(x)=\prod_{i \in V_{i}}\left(x-\theta^{i}\right) .
$$

Proposition 19: If $q \in V_{0}$ and $q \bmod n_{i} \in D_{0}^{\left(n_{i}\right)}$ for each $i \in\{1,2\}$, we have $v_{i}(x) \in \operatorname{GF}(q)[x]$ and $d_{i}^{\left(n_{j}\right)}(x) \in \operatorname{GF}(q)[x]$, and

$$
x^{n}-1=(x-1) d_{0}^{\left(n_{1}\right)}(x) d_{0}^{\left(n_{2}\right)}(x) v_{0}(x) d_{1}^{\left(n_{1}\right)}(x) d_{1}^{\left(n_{2}\right)}(x) v_{1}(x) .
$$

Proof: The proof of Proposition 3 is easily modified into a proof for this proposition.

Under the conditions that $q \in V_{0}$ and $q \bmod n_{i} \in D_{0}^{\left(n_{i}\right)}$ for each $i \in\{1,2\}$, let $\mathcal{V}_{(i, j, h)}^{\left(n_{1}, n_{2}, q\right)}$ denote the cyclic code over $\operatorname{GF}(q)$ with generator polynomial $v_{i}(x) d_{j}^{\left(n_{1}\right)}(x) d_{h}^{\left(n_{2}\right)}(x)$, where $(i, j, h) \in\{0,1\}^{3}$. The eight codes clearly have length $n$ and dimension $(n+1) / 2$. 
Proposition 20: There is an $\ell \in V_{1}$ such that $\ell \bmod n_{i} \in D_{1}^{\left(n_{i}\right)}$ for all $i \in\{1,2\}$.

Proof: It is easily checked that $g$ is such an element.

Due to Proposition 20, the proof of Theorem 12 works also for the following theorem.

Theorem 21: For each $(i, j, h) \in\{0,1\}^{3}$ the code $\mathcal{V}_{(i, j, h)}^{\left(n_{1}, n_{2}, q\right)}$ has parameters $[n,(n+1) / 2]$. Let $d_{(i, j, h)}$ denote the minimum odd-like weight in $\mathcal{V}_{(i, j, h)}^{\left(n_{1}, n_{2}, q\right)}$. Then

- $d_{(i, j, h)} \geq \sqrt{n}$, and

- $d_{(i, j, h)}^{2}-d_{(i, j, h)}+1 \geq n$ if $n_{1} \equiv-1 \quad(\bmod 8)$ and $n_{2} \equiv-1 \quad(\bmod 8)$.

\section{The binary case}

The following proposition will be useful later.

Proposition 22: Assume that $n_{1} \equiv \pm 1 \quad(\bmod 8)$ and $n_{2} \equiv \pm 1 \quad(\bmod 8)$. Then $-1 \in V_{1}$ and $-1 \in$ $D_{1}^{\left(n_{j}\right)}$ for all $j$ if and only if $n_{j} \equiv-1 \quad(\bmod 8)$ for all $j$.

Proof: Let $-1=g^{s} \nu^{i}$ for some fixed $0 \leq s \leq e-1$ and $0 \leq i \leq d-1$. By definition $-1 \in V_{1}$ if and only if $s+i$ is odd. By (2),

$$
-1 \equiv g^{s+i} \quad\left(\bmod n_{1}\right), \quad-1 \equiv g^{s} \quad\left(\bmod n_{2}\right) .
$$

The desired conclusion then follows.

We will need the following proposition in the sequel.

Proposition 23: The integer $2 \in V_{0}$ and $2 \in D_{0}^{\left(n_{j}\right)}$ for all $j$ if and only if $n_{j} \equiv \pm 1 \quad(\bmod 8)$ for all $j$.

Proof: Recall that $\mathbf{Z}_{n}^{*}=V_{0} \cup V_{1}$. By Proposition 1, there are two integers $0 \leq s \leq e-1$ and $0 \leq i \leq d-1$ such that $2=g^{s} \nu^{i}$. It then follows from (1) that

$$
g^{s+i} \equiv 2 \quad\left(\bmod n_{1}\right) \text { and } g^{s} \equiv 2 \quad\left(\bmod n_{2}\right) .
$$

Hence, $s$ is even if and only if $n_{2} \equiv \pm 1 \quad(\bmod 8)$. Note that $2 \in V_{0}$ if and only if $s+i$ is even. The desired conclusion then follows.

If $n_{1} \equiv \pm 1 \quad(\bmod 8)$ and $n_{2} \equiv \pm 1 \quad(\bmod 8)$, then we have the following factorization of $x^{n}-1$ over $\operatorname{GF}(2)$ :

$$
x^{n}-1=(x-1) d_{0}^{\left(n_{1}\right)}(x) d_{0}^{\left(n_{2}\right)}(x) v_{0}(x) d_{1}^{\left(n_{1}\right)}(x) d_{1}^{\left(n_{2}\right)}(x) v_{1}(x),
$$

and thus the eight binary cyclic codes $\mathcal{V}_{(i, j, h)}^{\left(n_{1}, n_{2}, 2\right)}$.

Example 7: Let $\left(n_{1}, n_{2}, q\right)=(7,17,2)$. In this case we have

$$
\begin{aligned}
& V_{0}=\left\{\begin{array}{l}
1,2,4,8,9,11,15,16,18,22,23,25,29,30,32,36,37,39,43,44,46,50,53,57,58,60, \\
64,65,67,71,72,74,78,79,81,86,88,92,93,95,99,100,106,107,109,113,114,116
\end{array}\right\}, \\
& V_{1}=\left\{\begin{array}{l}
3,5,6,10,12,13,19,20,24,26,27,31,33,38,40,41,45,47,48,52,54,55,59,61,62,66, \\
69,73,75,76,80,82,83,87,89,90,94,96,97,101,103,104,108,110,111,115,117,118
\end{array}\right\}
\end{aligned}
$$

and

$$
\begin{aligned}
d_{0}^{\left(n_{1}\right)}(x)= & x^{3}+x+1 \\
d_{1}^{\left(n_{1}\right)}(x)= & x^{3}+x^{2}+1 \\
d_{0}^{\left(n_{2}\right)}(x)= & x^{8}+x^{7}+x^{6}+x^{4}+x^{2}+x+1 \\
d_{1}^{\left(n_{2}\right)}(x)= & x^{8}+x^{5}+x^{4}+x^{3}+1 \\
v_{0}(x)= & x^{48}+x^{47}+x^{46}+x^{44}+x^{41}+x^{40}+x^{39}+x^{37}+x^{34}+x^{33}+x^{32}+x^{30}+x^{27}+x^{26}+ \\
& x^{25}+x^{23}+x^{20}+x^{19}+x^{18}+x^{16}+x^{14}+x^{11}+x^{10}+x^{9}+x^{7}+x^{4}+x^{3}+x^{2}+1 \\
v_{1}(x)= & x^{48}+x^{46}+x^{45}+x^{44}+x^{41}+x^{39}+x^{38}+x^{37}+x^{34}+x^{32}+x^{30}+x^{29}+x^{28}+x^{25}+ \\
& x^{23}+x^{22}+x^{21}+x^{18}+x^{16}+x^{15}+x^{14}+x^{11}+x^{9}+x^{8}+x^{7}+x^{4}+x^{2}+x+1 .
\end{aligned}
$$


The eight binary cyclic codes and their minimum weights are depicted in Table IX All the eight binary cyclic codes defined by the generalized cyclotomy of order two in this section have poor minimum weights. However, this does not mean that the new generalized cyclotomy of order two is not interesting in coding theory. In the sequel, we will see that some of the quaternary cyclic codes based on this new cyclotomy are the best cyclic codes.

TABLE IX

THE BINARY CYCLIC CODES OF LENGTH 119 AND DIMENSION 60 FROM THE NEW CYCLOTOMY

\begin{tabular}{|l|r|}
\hline Generator Polynomial & Minimum Weight \\
\hline \hline $\mathcal{V}_{(0,0,0)}^{(7,17,2)}$ & 8 \\
\hline $\mathcal{V}_{(1,0,0)}^{(7,17,2)}$ & 4 \\
\hline $\mathcal{V}_{(0,1,0)}^{(7,17,2)}$ & 4 \\
\hline $\mathcal{V}_{(0,0,1)}^{(7,17,2)}$ & 8 \\
\hline $\mathcal{V}_{(1,1,0)}^{(7,17,2)}$ & 8 \\
\hline $\mathcal{V}_{(1,0,1)}^{(7,17,2)}$ & 4 \\
\hline $\mathcal{V}_{(0,1,1)}^{(7,17,2)}$ & 4 \\
\hline $\mathcal{V}_{(1,1,1)}^{(7,17,2)}$ & 8 \\
\hline
\end{tabular}

\section{The ternary case}

The proof of Proposition 7 can be slightly modified into a proof of the following.

Proposition 24: The integer $3 \in D_{0}$ and $3 \bmod n_{i} \in D_{0}^{\left(n_{i}\right)}$ for all $i$ if and only if

$$
n_{1} \equiv \pm 1 \quad(\bmod 12) \text { and } n_{2} \equiv \pm 1 \quad(\bmod 12)
$$

Hence, in the case that $n_{1} \equiv \pm 1 \quad(\bmod 12)$ and $n_{2} \equiv \pm 1 \quad(\bmod 12)$, we have indeed the eight ternary cyclic codes $\mathcal{V}_{(i, j, h)}^{\left(n_{1}, n_{2}, 3\right)}$.

Example 8: When $\left(n_{1}, n_{2}, q\right)=(11,13,3)$, the minimum nonzero weights of the eight ternary codes are given in Table $\mathrm{X}$. Four of the eight codes have minimum weight 12, and the remaining four have minimum weight 6 .

TABLE X

THE TERNARY CYCLIC CODES OF LENGTH 143 AND DIMENSION 72 FROM THE CYCLOTOMY OF SECTIONV-A

\begin{tabular}{|l|r|}
\hline The code & Minimum Weight \\
\hline \hline $\mathcal{V}_{(0,0,0)}^{(11,13,3)}$ & 12 \\
\hline $\mathcal{V}_{(1,0,0)}^{(11,3)}$ & 6 \\
\hline $\mathcal{V}_{(0,1,0)}^{(11,13,3)}$ & 6 \\
\hline $\mathcal{V}_{(0,0,1)}^{(11,13,3)}$ & 12 \\
\hline $\mathcal{V}_{(1,1,0)}^{(11,13,3)}$ & 12 \\
\hline $\mathcal{V}_{(1,0,1)}^{(11,13)}$ & 6 \\
\hline $\mathcal{V}_{(0,1,1)}^{(11,13,3)}$ & 6 \\
\hline $\mathcal{V}_{(1,1,1)}^{(11,13,3)}$ & 12 \\
\hline
\end{tabular}




\section{E. The quaternary case}

The following proposition can be similarly proved.

Proposition 25: Assume that $n_{1} \equiv \pm 1 \quad(\bmod 4)$ and $n_{2} \equiv \pm 1 \quad(\bmod 4)$. Then $-1 \in V_{1}$ and $-1 \in$ $D_{1}^{\left(n_{j}\right)}$ for all $j$ if and only if $n_{j} \equiv-1 \quad(\bmod 4)$ for all $j$.

We will need the following proposition in the sequel whose proof is omitted here.

Proposition 26: The integer $4 \in V_{0}$ and $4 \bmod n_{j} \in D_{0}^{\left(n_{j}\right)}$ for all $j$ if $n_{j} \equiv \pm 1 \quad(\bmod 4)$ for all $j$.

If $n_{1} \equiv \pm 1 \quad(\bmod 4)$ and $n_{2} \equiv \pm 1 \quad(\bmod 4)$, then we have the following factorization of $x^{n}-1$ over $\mathrm{GF}(4)$ :

$$
x^{n}-1=(x-1) d_{0}^{\left(n_{1}\right)}(x) d_{0}^{\left(n_{2}\right)}(x) v_{0}(x) d_{1}^{\left(n_{1}\right)}(x) d_{1}^{\left(n_{2}\right)}(x) v_{1}(x),
$$

and thus the eight binary cyclic codes $\mathcal{V}_{(i, j, h)}^{\left(n_{1}, n_{2}, 4\right)}$.

Example 9: Let $\left(n_{1}, n_{2}, q\right)=(5,7,4)$. In this case the eight quaternary cyclic codes and their minimum weights are depicted in Table XI. Four of them are the best quaternary cyclic codes of length 35 and dimension 18 according to Table II. So this example shows that the new cyclotomy of order two is interesting in coding theory.

TABLE XI

THE QUATERNARY CYCLIC CODES OF LENGTH 35 AND DIMENSION 18 FROM THE NEW CYCLOTOMY

\begin{tabular}{|l|r|}
\hline Generator Polynomial & Minimum Weight \\
\hline \hline $\mathcal{V}_{(0,0,0)}^{(5,7,4)}$ & 8 \\
\hline $\mathcal{V}_{(1,0,0)}^{(5,7,4)}$ & 4 \\
\hline $\mathcal{V}_{(0,1,0)}^{(5,7,4)}$ & 4 \\
\hline $\mathcal{V}_{(0,7,4)}^{(5,4,4)}$ & 8 \\
\hline $\mathcal{V}_{(1,1,0)}^{(5,7,4)}$ & 8 \\
\hline $\mathcal{V}_{(1,0,1)}^{(5,7,4)}$ & 4 \\
\hline $\mathcal{V}_{(0,1,1)}^{(5,7,4)}$ & 4 \\
\hline $\mathcal{V}_{(1,1,1)}^{(5,7,4)}$ & 8 \\
\hline
\end{tabular}

\section{CONCLUDING REMARKS AND OPEN PROBLEMS}

Let $n$ be odd, and let $E_{0}$ and $E_{1}$ be two subsets of $\mathbf{Z}_{n} \backslash\{0\}=\{1,2, \cdots, n-1\}$. Let $\mu$ be an invertible element of $\mathbf{Z}_{n}$. A pair of sets $E_{0}$ and $E_{1}$, each of which is a union of nonzero $q$-cyclotomic cosets, forms a splitting of $n$ given by $\mu$ if

$$
\mu E_{0}=E_{1}, \mu E_{1}=E_{0}, E_{0} \cup E_{1}=\{1,2, \cdots, n-1\} .
$$

Clearly, if $E_{0}$ and $E_{1}$ is a splitting of $n$, we have

$$
\left|E_{0}\right|=\left|E_{1}\right|=(n-1) / 2 .
$$

As before, let $\theta$ be a $n$th primitive root of unity over an extension field of $\operatorname{GF}(q)$. Define a pair of polynomials

$$
g_{i}(x)=\prod_{j \in E_{i}}\left(x-\theta^{j}\right), j=0,1 .
$$

Since $E_{i}$ is the union of a number of $q$-cyclotomic cosets, each $g_{i}(x)$ must be over $\operatorname{GF}(q)$. The two codes of length $n$ over $\operatorname{GF}(q)$ with generator polynomials $g_{0}$ and $g_{1}$ are called a pair of duadic codes. The two codes of length $n$ over $\mathrm{GF}(q)$ with generator polynomials $(x-1) g_{0}(x)$ and $(x-1) g_{1}(x)$ are also called a pair of duadic codes. Duadic codes can also be defined in terms of idempotents, and include the quadratic residue codes. They were introduced and studied by Leon, Masley and Pless [13], Leon [14], Pless [18], 
and Pless, Masley and Leon [19], where a number of properties are described. Also all binary duadic codes of length until 241 are described in [19].

The cyclotomic cyclic codes presented in Sections IV and V] should be duadic codes (see [8], [7], [13], [14], [18], [19]). The contributions of Sections IV] and $\nabla$ are the extension of an earlier cyclotomy of order two, the new cyclotomy of order two, and the cyclotomic constructions of the cyclic codes over $\mathrm{GF}(q)$. According to [11, p. 233], there are four binary duadic codes of length 119 and dimension 60 and minimum weight 12 . It is interesting that all the best binary duadic codes of length 119 and dimension 60 are covered by the construction of Section IV-C.

The contribution of Section $\amalg$ iI the cyclotomic construction of the cyclic codes that contains some of the codes in [6] as special cases. Experimental data shows that all the cyclic codes over $\operatorname{GF}(q)$ obtained from the cyclotomy $\left(U_{0}, U_{1}\right)$ are the best or almost the best cyclic codes with the same length and dimension. So it would be interesting to further investigate this cyclotomic construction of cyclic codes. Note that the codes of Section III are not duadic, though they are cyclotomic.

Except for quadratic residue codes, it looks hard to develop general and tight lower bounds for duadic codes. The same looks true for the cyclotomic codes described in this paper. For specific cyclic codes obtained from the three constructions of this paper the $\mathrm{BCH}$ and other bounds described in [23], [22] may be employed.

In summary, all the three cyclotomic constructions described in this paper produce the best cyclic codes over certain fields $\mathrm{GF}(q)$, and are simple in structure. In addition, the extended cyclotomy and the new cyclotomy of order 2 may have applications in other areas.

Finally, we mention that the cyclic codes described in this paper can be employed to construct secret sharing schemes [2], authentication codes [9] and frequency hopping sequences [4].

\section{ACKNOWLEDGMENTS}

The author is very grateful to the anonymous reviewers and Dr. Mario Blaum for their comments and suggestions that improved the quality of this paper.

\section{REFERENCES}

[1] E. Betti and M. Sala, "A new bound for the minimum distance of a cyclic code from its defining set," IEEE Trans. Inform. Theory, vol. 52, no. 8, pp. 3700-3706, 2006.

[2] C. Carlet, C. Ding and J. Yuan, "Linear codes from highly nonlinear functions and their secret sharing schemes," IEEE Trans. Inform. Theory, vol. 51, no. 6, pp. 2089-2102, 2005.

[3] R. T. Chien, "Cyclic decoding procedure for the Bose-Chaudhuri-Hocquenghem codes," IEEE Trans. Inform. Theory, vol. IT-10, pp. 357-363, October 1964.

[4] C. Ding, R. Fuji-Hara, Y. Fujiwara, M. Jimbo, and M. Mishima, "Sets of frequency hopping sequences: bounds and optimal constructions," IEEE Trans. Inform. Theory, vol. 55, no. 7, pp. 3297-3304, July 2009.

[5] C. Ding and T. Helleseth, "New generalized cyclotomy and its applications," IEEE Finite Fields and Their Applications, vol. 4, pp. 140-166, 1998.

[6] C. Ding and T. Helleseth, "Generalized cyclotomic codes of length $p_{1}^{e_{1}} p_{2}^{e_{2}} \cdots p_{t}^{e_{t}}$," IEEE Trans. Inform. Theory, vol. 45, no. 2, pp. 467-474, 1999.

[7] C. Ding and V. Pless, "Cyclotomy and duadic codes of prime lengths," IEEE Trans. Inform. Theory, vol. 45, no. 2, 453-466, 1999.

[8] C. Ding, K. Y. Lam and C. Xing, "Enumeration and construction of all duadic codes of length $p$," Fundamenta Informaticae, vol. 38, no. 1, pp. 149-161, 1999.

[9] C. Ding and X. Wang, "A coding theory construction of new systematic authentication codes," Theoretical Computer Science, vol. 330, no. 1, pp. 81-99, 2005.

[10] G. D. Forney, "On decoding BCH codes," IEEE Trans. Inform. Theory, vol. IT-11, pp. 549-557, October 1965.

[11] W. C. Huffman and V. Pless, Fundamentals of Error-Correcting Codes, Cambridge University Press, Cambridge, 2003.

[12] Y. Jia, S. Ling, C. Xing, "On self-dual cyclic codes over finite fields," IEEE Trans. Inform. Theory, vol. 57, no. 4, pp. 2243-2251, 2011.

[13] J. S. Leon, J. M. Masley, and V. Pless, “Duadic codes,” IEEE Trans. Inform. Theory, vol. IT-30, pp. 709-714, 1984.

[14] J. S. Leon, "A probabilistic algorithm for computing minimum weight of large error-correcting codes," IEEE Trans. Inform. Theory, vol. IT-34, pp. 1354-1359, 1998.

[15] F. J. MacWilliams, "Cyclotomic numbers, coding theory and orthogonal polynomials," Discrete Mathematics, vol. 3, pp. 133-151, 1972.

[16] M. Moisio, "Exponential sums, Gauss sums and cyclic codes,” PhD Thesis, Acta Univ. Oul. A 306, 1998. 
[17] M. Moisio and Keijo O. Väänänen, "Two recursive algorithms for computing the weight distribution of certain irreducible cyclic codes," IEEE Trans. Inform. Theory, vol. 45, no. 4, pp. 1244-1249, May 1999.

[18] V. Pless, "Q-codes," J. Comb. Theory, vol. A 43, pp. 258-276, 1986.

[19] V. Pless, J. M. Masley, and J. S. Leon, "On weights in duadic codes," J. Comb. Theory, vol. A 44, pp. 6-21, 1987.

[20] E. Prange, "Some cyclic error-correcting codes with simple decoding algorithms," Air Force Cambridge Research Center-TN-58-156, Cam- bridge, Mass., April 1958.

[21] C. Rong, T. Helleseth, "Use characteristic sets to decode cyclic codes up to actual minimum distance," In: London Mathematical Society Lecture Note Series 233, pp. 297-312, 1996.

[22] M. van Eupen and J. H. van Lint, "On the minimum distance of ternary cyclic codes," IEEE Trans. Inform. Theory, vol. 39, no. 2, pp. 409-416, March 1993.

[23] J. H. van Lint and R. M. Wilson, "On the minimum distance of cyclic codes," IEEE Trans. Inform. Theory, vol. 32, no. 1, pp. 23-40, Jan. 1986.

[24] A. L. Whiteman, “A family of difference sets," Illinois J. Math., vol. 6, pp. 107-121, 1962. 